Pathophysiology

of Haemostasis and Thrombosis
Pathophysiol Haemost Thromb 2005;34:55-57

DOI: $\underline{10.1159 / 000089925}$

\title{
Good Mathematical Practice: Simulation of the Hemostatic-Thrombotic Mechanism, a Powerful Tool but One That Must Be Used with Circumspection
}

\author{
H. Coenraad Hemker ${ }^{a}$ Fazoil I. Ataullakhanov ${ }^{\text {b, c, d }}$ \\ a Synapse bv, Cardiovascular Research Institute Maastricht (CARIM), Maastricht, The Netherlands; \\ ${ }^{b}$ Laboratory of Physical Biochemistry of Blood, National Research Center for Hematology, Russian Academy \\ of Medical Sciences, Moscow, ${ }^{C}$ Laboratory of Metabolic Modeling and Bioinformatics, Institute of Theoretical \\ and Experimental Biophysics, Pushchino, Moscow Region, and ${ }^{\mathrm{d}}$ Faculty of Physics, Moscow State University, \\ Leninskie Gory, Moscow, Russia
}

Modeling means replacing a complicated mechanism by a simpler one that is more easy to understand and/or to manipulate. The complexity of the hemostatic and thrombotic system (H\&TS) is such that it is impossible to understand without modeling. The Quick-time is a model of the downregulation of the H\&TS by oral anticoagulation or liver disease. The bleeding time is another model of another subsystem of the H\&TS, with another scope. This illustrates that models never are complete and that they do not need to be complete to be useful. Actually, the most complete models are usually also complex, time-consuming, expensive, and extremely difficult to interprete.

Mathematical models are of a special kind (for a review of mathematical and computer research in coagulation, see [1]). In the first place they are impressive and impenetrable to the noninitiated - and the initiated are few. The biological scientist as a rule is not familiar with this approach. As soon as a biochemical mechanism is more complicated than the Michaelis-Menten model of enzyme action, many life-scientists and clinicians tend to be turned off, which is a pity because mathematical modeling is not an aim in itself, but should prove its value in the confrontation with actual laboratory data and needs the biologists for its verification. This is one reason to broach mathematical modeling in a journal on patho- physiology. In the second place mathematical models are special in that there is hardly a limit to the complications that can be introduced. Until the sixties of the last century the numerical solution of the mathematical formula that describe 'stiff' differential equations was an unresolved problem. Stiff differential equations describe phenomena that play on widely different time scales. The Michaelis-Menten model is an example: there the enzyme-substrate complex forms at the millisecond scale, whereas the formation of the product occurs on the minute-, or even hour-scale. The development of variable step length techniques together with the increase of computer power on our desks has solved this problem in the meantime. Nowadays chemical systems of any reasonable complexity are readily simulated. The partial differential equations that resolve the development of concentration changes in time and space, and especially that consider flow of blood (which is, from a physical point of view, rather complex and heterogeneous non-Newtonian fluid), may still stress the capacity of our computing systems, though.

The possibility to simulate more and more complicated systems, indeed so complicated as to incorporate almost all of the known reactions of the clotting system, comes at a price. By complete analogy with experimental models, increasing complexity and completeness may

\section{KARGER \\ Fax +4161306 1234 E-Mail karger@karger.ch} www.karger.com
(C) 2005 S. Karger AG, Basel

$1424-8832 / 05 / 0343-0055 \$ 22.00 / 0$

Accessible online at: www.karger.com/pht
Prof. H. Coenraad Hemker

Synapse bv, Cardiovascular Research Institute Maastricht (CARIM) PO Box 616

NL-6200 Maastricht MD (The Netherlands)

E-Mail hc.hemker@thrombin.com 
lead to negative effects. The way in which a mathematical solution is found becomes a black box for the laboratory scientist and special efforts are needed to make sure that there is a reasonable connection between what you put into it (reaction mechanisms and parameters) and what you get out of it (relations that look like experimental results) - we will come back to that point.

In the third place mathematical simulation, fascinating though it is to the addicted, cannot be an aim in itself. In the end it should be rigorously proven to be relevant to the biological situation. Therefore, any simulation experiment should go through three stages. The first stage is the 'what if' stage, it is the most imaginative, not to say the artistic part. The investigator imagines a plausible reaction mechanism and asks the question: what if this mechanism were operative in reality, how would it respond to variations in its different parameters? How would a model of thrombus growth react to halving the concentration of prothrombin? Or to an increase of the flow velocity? The larger part of the articles in the present volume are dedicated to this fascinating game. They analyze and predict effects of flow [2-5], diffusion [4, 5], vessel wall thrombomodulin [4], and chemical inhibitors [3] on the coagulation processes (clotting initiation [3, 4], pattern formation [2], propagation [3-5], shear stress profile, and lysis [5]).

The next step is the confrontation with the reality of the 'wet' experiment: can the model indeed quantitatively explain the experimental data? Is it possible to fit the simulated curves to experimental results within the limits of experimental error? This step requires close cooperation between the biochemist and the mathematician, specialized in curve-fitting, a branch of numerical mathematics. For example, a paper by Jesty et al. [6] focuses on the activation threshold in the contact activation pathway: theory and experiments are combined to show that a system with an autoactivating enzyme (factor XIIa) in the presence of an irreversible inhibitor (antithrombin III) has an activation threshold. An example of fruitful combination of stochastic simulation and thrombin generation experiments can also be found in the study by Lo et al. [7].

Lack of agreement between experimental and simulated data can be an important source of information in itself, indicating some gaps in our knowledge of the studied system, as is also illustrated by the study [7].

Only after an acceptable fit between the model and experimental data is obtained, comes the apex stone of the construction, the most crucial part and consolidation of the enterprise, because it then remains to be proven that the postulated mechanism is indeed operative in experimental reality. It may come as an unpleasant surprise, but it is quite possible that the same experimental curve can be explained by a large series of equally plausible mechanisms. A fit between experimental and simulated data does not at all mean that the assumptions underlying the simulation are right!

As soon as the number of parameters that are used to characterize the model system is much larger than the number of parameters that characterize the output, there usually are multiple ways to achieve the same fit. For example, the thrombin generation curve rises and falls after a given lag time. This type of relatively simple curve can be described by less than ten parameters. In a realistic simulation experiment it is the result of the complete clotting mechanism, which needs some eighty concentrations and reaction constants to be defined. It stands to reason that there are multiple ways to use eighty input parameters to get ten output parameters. Multiple variations of the reaction mechanism, but also multiple sets of constants within the same reaction mechanism will yield the same result. Decreasing the prothrombin content by $25 \%$ in a thrombin generation experiment can be compensated for by decreasing the antithrombin content as well. Two curves can result that are indistinguishable. Of course, it is possible to perform numerous experiments under various conditions (titrating tissue factor, prothrombin, antithrombin III, etc.) using a single set of model parameters and to obtain agreement between the model and multiple experimental curves. Actually, this way of validation is the one that should be and, sometimes, is used, but it still does not completely guarantee validity of the model. The number of fits is always finite, and the majority of system parameters cannot be experimentally changed for such verification (e.g., we can change prothrombin concentration to test whether it is accurately included in the model but it is very hard to change a prothrombin activation constant).

In this stage of simulation it is therefore good mathematical practice to investigate how far a solution that is found is indeed unique. If the curve fitting is done in a professional way, the outcome will not be a set of parameters by which the system will yield a fitting curve, but a set of multifolds, formulas that describe combinations of parameters that all will give a fitting curve.

In hemostasis and thrombosis, mechanisms that are realistic are so large that they seldom are unique, and a lot of reasonable alternatives are usually possible. Mechanisms that are unique are so simple that they are not realistic, just as the Michaelis-Menten model of enzyme 
action is much simpler than the catalytic mechanisms that we know to be operative, even in the simple conversion of one simple substrate by a simple enzyme.

It is an old saying among mathematicians that you can fit an elephant with five parameters and make it wig its tail with the sixth. The science of simulation can be readily used to simulate science. This is not to say that serious simulation is not a valuable tool. It only says that its use requires good mathematical practice and, in the end, profound confrontation of the data from the experiments 'in silico' and 'in solutio'.

\section{Conclusion}

Simulation comes in three steps: first, imagination of a suitable system; second, confrontation with the reality of experimental outcome; and third, consolidation of the conclusions by exclusion of alternatives. Together, this is a formidable task when the system is as large as the H\&TS is. This task requires close cooperation between the mathematically inclined and the laboratory. The articles in this volume are an important step in achieving such cooperation.

\section{References}

1 Ataullakhanov FI, Panteleev MA: Mathematical modeling and computer simulation in blood coagulation. Pathophysiol Haemost Thromb 2005;34:60-70.

2 Lobanov AI, Starozhilova TK: The effect of convective flows on blood coagulation processes. Pathophysiol Haemost Thromb 2005;34: 121-134.

3 Fogelson AL, Tania N: Coagulation under flow: the influence of flow-mediated transport on the initiation and inhibition of coagulation. Pathophysiol Haemost Thromb 2005;34:91108.

-4 Ermakova EA, Panteleev MA, Shnol EE: Blood coagulation and propagation of autowaves in flow. Pathophysiol Haemost Thromb 2005; 34:135-142.
5 Anand M, Rajagopal K, Rajagopal KR: A model for the formation and lysis of blood clots. Pathophysiol Haemost Thromb 2005; 34:109-120.

-6 Jesty J, Rodriguez J, Beltrami E: Demonstration of a threshold response in a proteolytic feedback system: control of the autoactivation of factor XII. Pathophysiol Haemost Thromb 2005;34:71-79.

$>7$ Lo K, Denney WS, Diamond SL: Stochastic modeling of blood coagulation initiation. Pathophysiol Haemost Thromb 2005;34:8090. 